\title{
Robust and Efficient Optimization Using a Marquardt-Levenberg Algorithm with R Package marqLevAlg
}

by Viviane Philipps, Boris P. Hejblum, Mélanie Prague, Daniel Commenges and Cécile Proust-Lima

\begin{abstract}
Implementations in R of classical general-purpose algorithms for local optimization generally have two major limitations which cause difficulties in applications to complex problems: too loose convergence criteria and too long calculation time. By relying on a Marquardt-Levenberg algorithm (MLA), a Newton-like method particularly robust for solving local optimization problems, we provide with marqLevAlg package an efficient and general-purpose local optimizer which (i) prevents convergence to saddle points by using a stringent convergence criterion based on the relative distance to minimum/maximum in addition to the stability of the parameters and of the objective function; and (ii) reduces the computation time in complex settings by allowing parallel calculations at each iteration. We demonstrate through a variety of cases from the literature that our implementation reliably and consistently reaches the optimum (even when other optimizers fail) and also largely reduces computational time in complex settings through the example of maximum likelihood estimation of different sophisticated statistical models.
\end{abstract}

\section{Introduction}

Optimization is an essential task in many computational problems. In statistical modeling, for instance, in the absence of analytical solutions, maximum likelihood estimators are often retrieved using iterative optimization algorithms, which locally solve the problem from given starting values.

Steepest descent algorithms are among the most famous general local optimization algorithms. They generally consist in updating parameters according to the steepest gradient (gradient descent) possibly scaled by the Hessian in the Newton (Newton-Raphson) algorithm or an approximation of the Hessian based on the gradients in the quasi-Newton algorithms (e.g., Broyden-Fletcher-GoldfarbShanno - BFGS). Newton-like algorithms have been shown to provide good convergence properties (Joe and Nash, 2003) and were demonstrated in particular to behave better than Expectation-Maximization (EM) algorithms in several contexts of Maximum Likelihood Estimation, such as the random-effect models (Lindstrom and Bates, 1988) or the latent class models (Proust and Jacqmin-Gadda, 2005). Among Newton methods, the Marquardt-Levenberg algorithm, initially proposed by Levenberg (Levenberg, 1944), then Marquardt (Marquardt, 1963), combines BFGS and gradient descent methods to provide a more robust optimization algorithm. Like other Newton methods, the MarquardtLevenberg algorithm is designed to find a local optimum of the objective function from given initial values. When dealing with multimodal objective functions, it can thus converge to local optimum and needs to be combined with a grid search to retrieve the global optimum.

The R software includes multiple solutions for local and global optimization tasks (see CRAN task View on Optimization (Theussl et al., 2014)). In particular, the optim function in base R offers different algorithms for general-purpose optimization, and so does optimx, a more recent package extending optim (Nash and Varadhan, 2011). Numerous additional packages are available for different contexts, from nonlinear least square problems (including some exploiting Marquardt-Levenberg idea like minpack.1m (Elzhov et al., 2016) and nlmrt (Nash, 2016)) to stochastic optimization and algorithms based on the simplex approach. However, R software could benefit from a general-purpose $\mathrm{R}$ implementation of the Marquardt-Levenberg algorithm.

We present here an $\mathrm{R}$ implementation of the Marquardt-Levenberg algorithm in the package marqLevAlg, which relies on a stringent convergence criterion based on the first and second derivatives to avoid loosely convergence (Prague et al., 2012) and includes (from version 2.0.1) parallel computations within each iteration to speed up convergence. This implementation is particularly dedicated to complex settings, that is, when a large number of parameters are optimized, and/or the computation of the objective function is time-consuming. The parallel computations speed up the procedure, and the stringent convergence criterion prevents false convergences on the flat regions of the objective function obtained with convergence criteria based on the function stability.

Section 2 and 3 describe the algorithm and the implementation, respectively. Then Section 4 provides an example of a call with the estimation of a linear mixed model. A benchmark of the package is reported in Section 5 with the performances of parallel implementation. Performances of Marquardt-Levenberg algorithm implementation are also challenged in Section 6 using a variety of simple and complex examples from the literature and compared with other optimizers. Finally, 
Section 7 concludes.

\section{Methodology}

\section{The Marquardt-Levenberg algorithm}

The Marquardt-Levenberg algorithm (MLA) can be used for any problem where a function $\mathcal{F}(\theta)$ has to be minimized (or equivalently, function $\mathcal{L}(\theta)=-\mathcal{F}(\theta)$ has to be maximized) according to a set of $m$ unconstrained parameters $\theta$ as long as the second derivatives of $\mathcal{F}(\theta)$ exist. In statistical applications, for instance, the objective function is the deviance to be minimized or the log-likelihood to be maximized.

Our improved MLA iteratively updates the vector $\theta^{(k)}$ from a starting point $\theta^{(0)}$ until convergence using the following formula at iteration $k+1$ :

$$
\theta^{(k+1)}=\theta^{(k)}-\delta_{k}\left(\tilde{H}\left(\mathcal{F}\left(\theta^{(k)}\right)\right)\right)^{-1} \nabla\left(\mathcal{F}\left(\theta^{(k)}\right)\right),
$$

where $\theta^{(k)}$ is the set of parameters at iteration $k, \nabla\left(\mathcal{F}\left(\theta^{(k)}\right)\right)$ is the gradient of the objective function at iteration $k$, and $\tilde{H}\left(\mathcal{F}\left(\theta^{(k)}\right)\right)$ is the Hessian matrix $H\left(\mathcal{F}\left(\theta^{(k)}\right)\right)$ where the diagonal terms are replaced by $\tilde{H}\left(\mathcal{F}\left(\theta^{(k)}\right)\right)_{i i}=H\left(\mathcal{F}\left(\theta^{(k)}\right)\right)_{i i}+\lambda_{k}\left[\left(1-\eta_{k}\right)\left|H\left(\mathcal{F}\left(\theta^{(k)}\right)\right)_{i i}\right|+\eta_{k} \operatorname{tr}\left(H\left(\mathcal{F}\left(\theta^{(k)}\right)\right)\right)\right]$. In the original MLA, the Hessian matrix is inflated by a scaled identity matrix. Following Fletcher (1971), we consider refined inflation based on the curvature. The diagonal inflation of our improved MLA makes it an intermediate between the steepest descent method and the Newton method. The parameters $\delta_{k}, \lambda_{k}$, and $\eta_{k}$ are scalars specifically determined at each iteration $k$. Parameter $\delta_{k}$ is fixed to 1 unless the objective function is not reduced, in which case a line search determines the locally optimal step length. Parameters $\lambda_{k}$ and $\eta_{k}$ are internally modified in order to ensure that (i) $\tilde{H}\left(\mathcal{F}\left(\theta^{(k)}\right)\right)$ be definite-positive at each iteration $k$, and (ii) $\tilde{H}\left(\mathcal{F}\left(\theta^{(k)}\right)\right)$ approaches $H\left(\mathcal{F}\left(\theta^{(k)}\right)\right)$ when $\theta^{(k)}$ approaches $\hat{\theta}$.

When the problem encounters a unique solution, the minimum is reached whatever the chosen initial values.

\section{Stringent convergence criteria}

As in any iterative algorithm, the convergence of MLA is achieved when convergence criteria are fulfilled. In marqLevAlg package, convergence is defined according to three criteria:

- parameters stability: $\sum_{j=1}^{m}\left(\theta_{j}^{(k+1)}-\theta_{j}^{(k)}\right)^{2}<\epsilon_{a}$;

- objective function stability: $\left|\mathcal{F}^{(k+1)}-\mathcal{F}^{(k)}\right|<\epsilon_{b}$;

- relative distance to minimum/maximum (RDM): $\frac{\nabla\left(\mathcal{F}\left(\theta^{(k)}\right)\right)\left(H\left(\mathcal{F}\left(\theta^{(k)}\right)\right)\right)^{-1} \nabla\left(\mathcal{F}\left(\theta^{(k)}\right)\right)}{m}<\epsilon_{d}$.

The original Marquardt-Levenberg algorithm (Marquardt, 1963) and its implementations (Elzhov et al., 2016; Nash, 2016) consider the two first criteria (as well as a third one based on the angle between the objective function and its gradient). Yet, these criteria, which are also used in many other iterative algorithms, do not ensure convergence towards an actual optimum. They only ensure the convergence towards a saddle point. We thus chose to complement the parameter and objective function stability by the relative distance to minimum/maximum. As it requires the Hessian matrix to be invertible, it prevents any convergence to a saddle point and is thus essential to ensure that an optimum is truly reached. When the Hessian is not invertible, RDM is set to $1+\epsilon_{d}$, and convergence criteria cannot be fulfilled.

Although it constitutes a relevant convergence criterion in any optimization context, RDM was initially designed for log-likelihood maximization problems, that is, cases where $\mathcal{F}(\theta)=-\mathcal{L}(\theta)$ with $\mathcal{L}$ the log-likelihood. In that context, RDM can be interpreted as the ratio between the numerical error and the statistical error (Commenges et al., 2006, Prague et al. (2013)).

The three thresholds $\epsilon_{a}, \epsilon_{b}$, and $\epsilon_{d}$ can be adjusted, but values around 0.0001 are usually sufficient to guarantee a correct convergence. In some complex log-likelihood maximization problems, for instance, Prague et al. (2013) showed that the RDM convergence properties remain acceptable, providing $\epsilon_{d}$ is below 0.1 (although the lower, the better). 


\section{Derivatives calculation}

MLA update relies on first $\left(\nabla\left(\mathcal{F}\left(\theta^{(k)}\right)\right)\right)$ and second $\left(H\left(\mathcal{F}\left(\theta^{(k)}\right)\right)\right)$ derivatives of the objective function $\mathcal{F}\left(\theta^{(k)}\right)$ at each iteration $\mathrm{k}$. The gradient and the Hessian may sometimes be calculated analytically, but numerical approximation can become necessary in a general framework. In marqLevAlg package, in the absence of analytical gradient computation, the first derivatives are computed by central finite differences. In the absence of analytical Hessian, the second derivatives are computed using forward finite differences. The step of finite difference for each derivative depends on the value of the involved parameter. It is set to $\max \left(10^{-7}, 10^{-4}\left|\theta_{j}\right|\right)$ for parameter $j$.

When both the gradient and the Hessian are to be numerically computed, numerous evaluations of $\mathcal{F}$ are required at each iteration:

- $2 \times m$ evaluations of $\mathcal{F}$ for the numerical approximation of the gradient function;

- $\frac{m \times(m+1)}{2}$ evaluations of $\mathcal{F}$ for the numerical approximation of the Hessian matrix.

The number of derivatives thus grows quadratically with the number $m$ of parameters, and calculations are per se independent as done for different vectors of parameters $\theta$.

When the gradient is analytically calculated, only the second derivatives have to be approximated, requiring $2 \times m$ independent calls to the gradient function. In that case, the complexity thus linearly increases with $m$.

In both cases, and especially when each calculation of derivative is long and/or $m$ is large, parallel computations of independent $\mathcal{F}$ evaluations become particularly relevant to speed up the estimation process.

\section{Special case of a log-likelihood maximization}

When the optimization problem is the maximization of the log-likelihood $\mathcal{L}(\theta)$ of a statistical model according to parameters $\theta$, the Hessian matrix of the $\mathcal{F}(\theta)=-\mathcal{L}(\theta)$ calculated at the optimum $\hat{\theta}$, $\mathcal{H}(\mathcal{F}(\hat{\theta}))=-\left.\frac{\partial^{2} \mathcal{L}(\theta)}{\partial \theta^{2}}\right|_{\theta=\hat{\theta}}$, provides an estimator of the Fisher Information matrix. The inverse of $\mathcal{H}(\mathcal{F}(\hat{\theta}))$ computed in the package thus provides an estimator of the variance-covariance matrix of the optimized vector of parameters $\hat{\theta}$.

\section{Implementation}

\section{marqLevAlg function}

The call of the marqLevAlg function, or its shorcut mla, is the following :

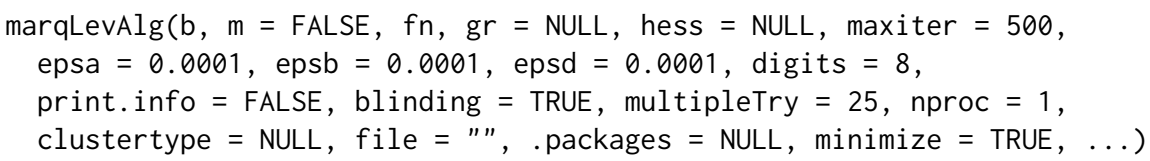

Argument $b$ is the set of initial parameters; alternatively, its length $m$ can be entered. $f n$ is the function to optimize; it should take the parameter vector as the first argument, and additional arguments are passed in .... Optional gr and hess refer to the functions implementing the analytical calculations of the gradient and the Hessian matrix, respectively. maxiter is the maximum number of iterations. Arguments epsa, epsb, and epsd are the thresholds for the three convergence criteria defined in Section 2.2.2. print. info specifies if details on each iteration should be printed; such information can be reported in a file if argument file is specified, and digits indicates the number of decimals in the eventually reported information during optimization. blinding is an option allowing the algorithm to go on even when the fn function returns NA, which is then replaced by the arbitrary value of 500,000 (for minimization) and -500,000 (for maximization). Similarly, if an infinite value is found for the chosen initial values, the multipleTry option will internally reshape $b$ (up to multipleTry times) until a finite value is got, and the algorithm can be correctly initialized. The parallel framework is first stated by the nproc argument, which gives the number of cores, and by the clustertype argument (see the next section). In the case where the fn function depends on $\mathrm{R}$ packages, these should be given as a character vector in the . packages argument. Finally, the minimize argument offers the possibility to minimize or maximize the objective function $\mathrm{fn}$; a maximization problem is implemented as the minimization of the opposite function $(-f n)$. 


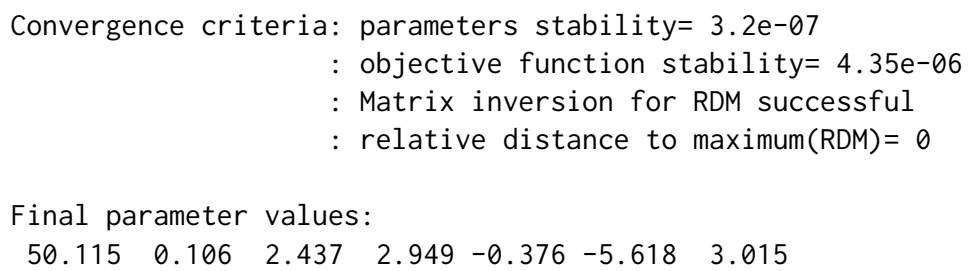

The printed output estim shows that the algorithm converged in 18 iterations with convergence criteria of $3.2 \mathrm{e}-07,4.35 \mathrm{e}-06$, and 0 for parameters stability, objective function stability, and RDM, respectively. The output also displays the list of coefficient values at the optimum. All this information can also be recovered in the estim object, where item $b$ contains the estimated coefficients.

As mentioned in Section 2.2.4, in log-likelihood maximization problems, the inverse of the Hessian given by the program provides an estimate of the variance-covariance matrix of the coefficients at the optimum. The upper triangular matrix of the inverse Hessian is thus systematically computed in object $\mathrm{v}$. When appropriate, the summary function can output this information with option loglik $=$ TRUE. With this option, the summary also includes the square root of these variances (i.e., the standards errors), the corresponding Wald statistic, the associated $p$-value, and the $95 \%$ confidence interval boundaries for each parameter:

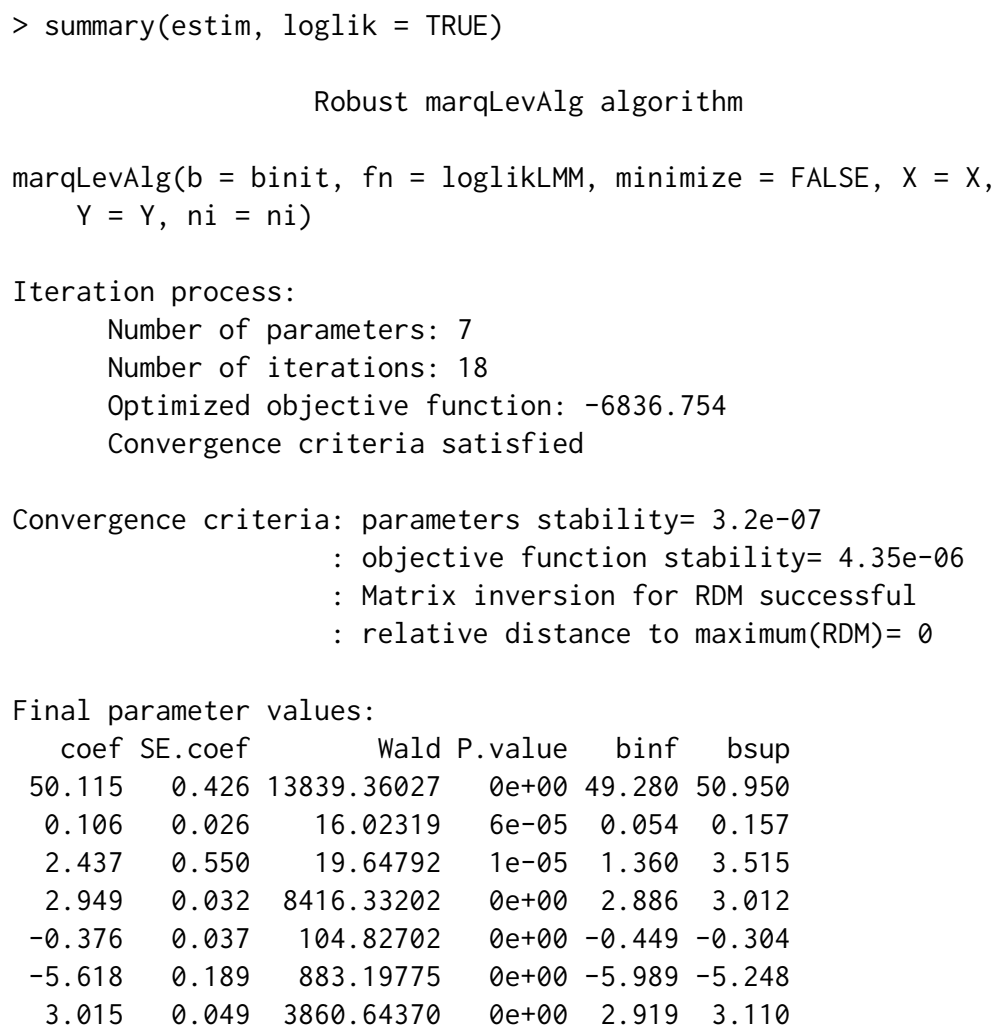

The exact same model can also be estimated in a parallel mode using FORK implementation of parallelism (here with two cores):

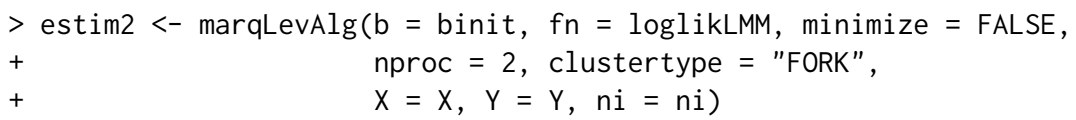

It can also be estimated by using analytical gradients (provided in gradient function gradLMM with the same arguments as loglikLMM):

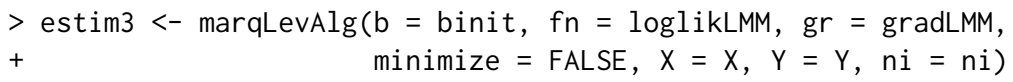

In all three situations, the program converges to the same maximum as shown in Table 1 for the estimation process and Table 2 for the parameter estimates. The iteration process is identical when 
using either the sequential or the parallel code (number of iterations, final convergence criteria, etc.). It necessarily differs slightly when using the analytical gradient, as the computation steps are not identical (e.g., here it converges in 15 iterations rather than 18), but all the final results are identical.

\begin{tabular}{lrrr}
\hline & Object estim & Object estim2 & Object estim3 \\
\hline Number of cores & 1 & 2 & 1 \\
Analytical gradient & $\mathrm{no}$ & $\mathrm{no}$ & yes \\
Objective Function & -6836.754 & -6836.754 & -6836.754 \\
Number of iterations & 18 & 18 & 15 \\
Parameter Stability & $3.174428 \mathrm{e}-07$ & $3.174428 \mathrm{e}-07$ & $6.633702 \mathrm{e}-09$ \\
Likelihood stability & $4.352822 \mathrm{e}-06$ & $4.352822 \mathrm{e}-06$ & $9.159612 \mathrm{e}-08$ \\
RDM & $1.651774 \mathrm{e}-12$ & $1.651774 \mathrm{e}-12$ & $2.935418 \mathrm{e}-17$ \\
\hline
\end{tabular}

Table 1: Summary of the estimation process of a linear mixed model using 'marqLevAlg' function run either in sequential mode with numerical gradient calculation (object estim), parallel mode with numerical gradient calculation (object estim2), or sequential mode with analytical gradient calculation (object estim3).

\begin{tabular}{lrrrrrr}
\hline & \multicolumn{2}{c}{ Object estim } & \multicolumn{2}{c}{ Object estim2 } & \multicolumn{2}{c}{ Object estim3 } \\
\hline & Coef & SE & Coef & SE & Coef & SE \\
\hline Parameter 1 & 50.1153 & 0.4260 & 50.1153 & 0.4260 & 50.1153 & 0.4260 \\
Parameter 2 & 0.1055 & 0.0264 & 0.1055 & 0.0264 & 0.1055 & 0.0264 \\
Parameter 3 & 2.4372 & 0.5498 & 2.4372 & 0.5498 & 2.4372 & 0.5498 \\
Parameter 4 & 2.9489 & 0.0321 & 2.9489 & 0.0321 & 2.9489 & 0.0321 \\
Parameter 5 & -0.3764 & 0.0368 & -0.3764 & 0.0368 & -0.3764 & 0.0368 \\
Parameter 6 & -5.6183 & 0.1891 & -5.6183 & 0.1891 & 5.6183 & 0.1891 \\
Parameter 7 & 3.0145 & 0.0485 & 3.0145 & 0.0485 & 3.0145 & 0.0485 \\
\hline
\end{tabular}

Table 2: Estimates (Coef) and standard error (SE) of the parameters of a linear mixed model fitted using 'marqLevAlg' function run either in sequential mode with numerical gradient calculation (object estim), parallel mode with numerical gradient calculation (object estim2), or sequential mode with analytical gradient calculation (object estim3).

\section{Benchmark}

We aimed at evaluating and comparing the performances of the parallelization in some time-consuming examples. We focused on three examples of sophisticated models from the mixed models area, estimated by maximum likelihood. These examples rely on packages using three different languages, thus illustrating the behavior of marqLevAlg package with a program exclusively written in R (JM, Rizopoulos (2010)) and programs, including Rcpp (CInLPN, Taddé et al. (2019)) and Fortran90 (lcmm, Proust-Lima et al. (2017)) languages, widely used in complex situations.

We first describe the generated dataset on which the benchmark has been realized. We then introduce each statistical model and associated program. Finally, we detail the results obtained with the three programs. Each time, the model has been estimated sequentially and with a varying number of cores in order to provide the program speed-up. We used a Linux cluster with 32 cores machines and 100 replicates to assess the variability. Codes and datasets used in this section are available at https://github.com/VivianePhilipps/marqLevAlgPaper.

\section{Simulated dataset}

We generated a dataset of 20,000 subjects having repeated measurements of a marker Ycens (measured at times t) up to a right-censored time of event tsurv with the indicator that the event occurred event. The data were generated according to a 4 latent class joint model (Proust-Lima et al., 2014). This model assumes that the population is divided into 4 latent classes, each class having a specific trajectory of the marker defined according to a linear mixed model with specific parameters, and specific risk of event defined according to a parametric proportional hazard model with specific parameters too. The 
class-specific linear mixed model included a basis of natural cubic splines with 3 equidistant knots taken at times 5, 10 and 15, associated with fixed and correlated random effects. The proportional hazard model included a class-specific Weibull risk adjusted on 3 covariates: one binary (Bernoulli with $50 \%$ probability) and two continous variables (standard Gaussian and Gaussian with mean 45 and standard deviation 8). The proportion of individuals in each class is about $22 \%, 17 \%, 34 \%$, and $27 \%$ in the sample.

Below are given the five first rows of the three first subjects:

\begin{tabular}{|c|c|c|c|c|c|c|c|c|}
\hline i & class & X1 & $\times 2$ & $\times 3$ & t & Ycens & tsurv & event \\
\hline 1 & 2 & 0 & 0.6472205 & 43.42920 & 0 & 61.10632 & 20.000000 & 0 \\
\hline 1 & 2 & 0 & 0.6472205 & 43.42920 & 1 & 60.76988 & 20.000000 & 0 \\
\hline 1 & 2 & 0 & 0.6472205 & 43.42920 & 2 & 58.72617 & 20.000000 & $\theta$ \\
\hline 1 & 2 & 0 & 0.6472205 & 43.42920 & 3 & 56.76015 & 20.000000 & $\theta$ \\
\hline 1 & 2 & $\theta$ & 0.6472205 & 43.42920 & 4 & 54.04558 & 20.000000 & 0 \\
\hline 2 & 1 & $\theta$ & 0.3954846 & 43.46060 & 0 & 37.95302 & 3.763148 & 1 \\
\hline 2 & 1 & 0 & 0.3954846 & 43.46060 & 1 & 34.48660 & 3.763148 & \\
\hline 2 & 1 & 0 & 0.3954846 & 43.46060 & 2 & 31.39679 & 3.763148 & 1 \\
\hline 2 & 1 & $\theta$ & 0.3954846 & 43.46060 & 3 & 27.81427 & 3.763148 & 1 \\
\hline 2 & 1 & $\theta$ & 0.3954846 & 43.46060 & 4 & NA & 3.763148 & 1 \\
\hline 3 & 3 & 0 & 1.0660837 & 42.08057 & 0 & 51.60877 & 15.396958 & 1 \\
\hline 3 & 3 & 0 & 1.0660837 & 42.08057 & 1 & 53.80671 & 15.396958 & 1 \\
\hline 3 & 3 & $\theta$ & 1.0660837 & 42.08057 & 2 & 51.11840 & 15.396958 & 1 \\
\hline & 3 & 0 & 1.0660837 & 42.08057 & 3 & 50.64331 & 15.396958 & 1 \\
\hline & 3 & 0 & 1.0660837 & 42.08057 & 4 & 50.87873 & 15.396958 & \\
\hline
\end{tabular}

\section{Statistical models}

\section{Joint shared random effect model for a longitudinal marker and a time to event: package JM}

The maximum likelihood estimation of joint shared random effect models has been made available in R with the JM package (Rizopoulos, 2010). The implemented optimization functions are optim and nlminb. We added the marqLevALg function for the purpose of this example. We considered a subsample of the simulated dataset, consisting of 5,000 randomly selected subjects.

The joint shared random effect model is divided into two submodels jointly estimated:

- a linear mixed submodel for the repeated marker $Y$ measured at different times $t_{i j}\left(j=1, \ldots, n_{i}\right)$ :

$$
\begin{aligned}
Y_{i}\left(t_{i j}\right) & =\tilde{Y}_{i}\left(t_{i j}\right)+\varepsilon_{i j} \\
& =X_{i}\left(t_{i j}\right) \beta+Z_{i}\left(t_{i j}\right) u_{i}+\varepsilon_{i j},
\end{aligned}
$$

where, in our example, $X_{i}(t)$ contained the intercept, the class indicator, the 3 simulated covariates, a basis of natural cubic splines on time $t$ (with 2 internal knots at times 5 and 15), and the interactions between the splines and the time-invariant covariates, resulting in 20 fixed effects. $Z_{i}(t)$ contained the intercept and the same basis of natural cubic splines on time $t$, and was associated with $u_{i}$, the 4 -vector of correlated Gaussian random effects. $\varepsilon_{i j}$ was the independent Gaussian error.

- a survival submodel for the right censored time-to-event:

$$
\alpha_{i}(t)=\alpha_{0}(t) \exp \left(X_{s i} \gamma+\eta \tilde{Y}_{i}(t)\right)
$$

where, in our example, the vector $X_{s i}$, containing the 3 simulated covariates, was associated with the vector of parameters $\gamma$; the current underlying level of the marker $\tilde{Y}_{i}(t)$ was associated with parameter $\eta$ and the baseline hazard $\alpha_{0}(t)$ was defined using a basis of B-splines with 1 interior knot.

The length of the total vector of parameters $\theta$ to estimate was 40 (20 fixed effects and 11 variance component parameters in the longitudinal submodel and 9 parameters in the survival submodel).

One particularity of this model is that the log-likelihood does not have a closed-form. It involves an integral over the random effects (here, of dimension 4), which is numerically computed using an adaptive Gauss-Hermite quadrature with 3 integration points for this example.

As package JM includes an analytical computation of the gradient, we ran two estimations: one with the analytical gradient and one with the numerical approximation to compare the speed up and execution times. 


\section{Latent class linear mixed model: package $1 \mathrm{cmm}$}

The second example is a latent class linear mixed model, as implemented in the hlme function of the $1 \mathrm{cmm} \mathrm{R}$ package. The function uses a previous implementation of the Marquardt algorithm coded in Fortran 90 and in sequential mode. For the purpose of this example, we extracted the log-likelihood computation programmed in Fortran90 to be used with marqLevAlg package.

The latent class linear mixed model consists of two submodels estimated jointly:

- a multinomial logistic regression for the latent class membership $\left(c_{i}\right)$ :

$$
\mathbb{P}\left(c_{i}=g\right)=\frac{\exp \left(W_{i} \zeta_{g}\right)}{\sum_{l=1}^{G} \exp \left(W_{i} \zeta_{l}\right)} \quad, \quad \text { with } g=1, \ldots, G,
$$

where $\zeta_{G}=0$ for identifiability, and $W_{i}$ contained an intercept and the 3 covariates.

- a linear mixed model specific to each latent class $g$ for the repeated outcome $Y$ measured at times $t_{i j}\left(j=1, \ldots, n_{i}\right)$ :

$$
Y_{i}\left(t_{i j} \mid c_{i}=g\right)=X_{i}\left(t_{i j}\right) \beta_{g}+Z_{i}\left(t_{i j}\right) u_{i g}+\varepsilon_{i j},
$$

where, in this example, $X_{i}(t)$ and $Z_{i}(t)$ contained an intercept, time $t$, and quadratic time. The vector $u_{i g}$ of correlated Gaussian random effects had a proportional variance across latent classes, and $\varepsilon_{i j}$ were independent Gaussian errors.

The log-likelihood of this model has a closed-form, but it involves the logarithm of a sum over latent classes, which can become computationally demanding. We estimated the model on the total sample of 20,000 subjects with 1,2,3, and 4 latent classes, which corresponded to 10, 18, 26, and 34 parameters to estimate, respectively.

\section{Multivariate latent process mixed model: package CInLPN}

The last example is provided by the CInLPN package, which relies on the Rcpp language. The function fits a multivariate linear mixed model combined with a system of difference equations in order to retrieve temporal influences between several repeated markers (Taddé et al., 2019). We used the data example provided in the package where three continuous markers $L_{-} 1, L_{-} 2, L_{-} 3$ were repeatedly measured over time. The model related each marker $k(k=1,2,3)$ measured at observation times $t_{i j k}$ $(j=1, \ldots, T)$ to its underlying level $\Lambda_{i k}\left(t_{i j k}\right)$ as follows:

$$
\mathrm{L}_{i k}\left(t_{i j k}\right)=\eta_{0 k}+\eta_{1 k} \Lambda_{i k}\left(t_{i j k}\right)+\epsilon_{i j k}
$$

where $\epsilon_{i j k}$ are independent Gaussian errors and $\left(\eta_{0}, \eta_{1}\right)$ parameters to estimate. Simultaneously, the structural model defines the initial state at time $0\left(\Lambda_{i k}(0)\right)$ and the change over time at subsequent times $t$ with $\delta$ is a discretization step:

$$
\begin{aligned}
\Lambda_{i k}(0) & =\beta_{0 k}+u_{i k} \\
\frac{\Lambda_{i k}(t+\delta)-\Lambda_{i k}(t)}{\delta} & =\gamma_{0 k}+v_{i k}+\sum_{l=1}^{K} a_{k l} \Lambda_{i l}(t),
\end{aligned}
$$

where $u_{i k}$ and $v_{i k}$ are Gaussian random effects.

Again, the log-likelihood of this model that depends on 27 parameters has a closed-form, but it may involve complex calculations.

\section{Results}

All the models have been estimated with 1, 2, 3, 4, 6, 8, 10, 15, 20, 25, and 30 cores. To fairly compare the execution times, we ensured that changing the number of cores did not affect the final estimation point or the number of iterations needed to converge. The mean of the speed up over the 100 replicates is reported in Table 3 and plotted in Figure 1.

The joint shared random effect model (JM) converged in 16 iterations after 4279 seconds in sequential mode when using the analytical gradient. Running the algorithm in parallel on 2 cores made the execution 1.85 times shorter. Computational time was gradually reduced with a number of cores 


\begin{tabular}{lrrrrrrr}
\hline & \multicolumn{2}{c}{ JM } & \multicolumn{3}{c}{ hlme } & & CInLPN \\
\hline & analytic & numeric & G=1 & G=2 & G=3 & G=4 \\
\hline Number of parameters & 40 & 40 & 10 & 18 & 26 & 34 & 27 \\
Number of iterations & 16 & 16 & 30 & 30 & 30 & 30 & 13 \\
Number of elements in foreach loop & 40 & 860 & 65 & 189 & 377 & 629 & 405 \\
Sequential time (seconds) & 4279 & 14737 & 680 & 3703 & 10402 & 22421 & 272 \\
Speed up with 2 cores & 1.85 & 1.93 & 1.78 & 1.93 & 1.94 & 1.96 & 1.89 \\
Speed up with 3 cores & 2.40 & 2.80 & 2.35 & 2.81 & 2.88 & 2.92 & 2.75 \\
Speed up with 4 cores & 2.97 & 3.57 & 2.90 & 3.58 & 3.80 & 3.87 & 3.56 \\
Speed up with 6 cores & 3.66 & 4.90 & 3.49 & 5.01 & 5.44 & 5.66 & 4.95 \\
Speed up with 8 cores & 4.15 & 5.84 & 3.71 & 5.84 & 6.90 & 7.26 & 5.96 \\
Speed up with 10 cores & 4.23 & 6.69 & 3.98 & 6.70 & 8.14 & 8.96 & 6.89 \\
Speed up with 15 cores & 4.32 & 7.24 & 3.59 & 7.29 & 10.78 & 12.25 & 8.14 \\
Speed up with 20 cores & 4.28 & 7.61 & 3.11 & 7.71 & 12.00 & 15.23 & 8.36 \\
Speed up with 25 cores & 3.76 & 7.29 & 2.60 & 7.37 & 12.30 & 16.84 & 8.11 \\
Speed up with 30 cores & 3.41 & 6.82 & 2.47 & 6.82 & 13.33 & 17.89 & 7.83 \\
\hline
\end{tabular}

Table 3: Estimation process characteristics for the 3 different programs (JM, hlme, and CInLPN). Analytic and Numeric refer to the analytical and numerical computations of the gradient in JM; G refers to the number of latent classes.
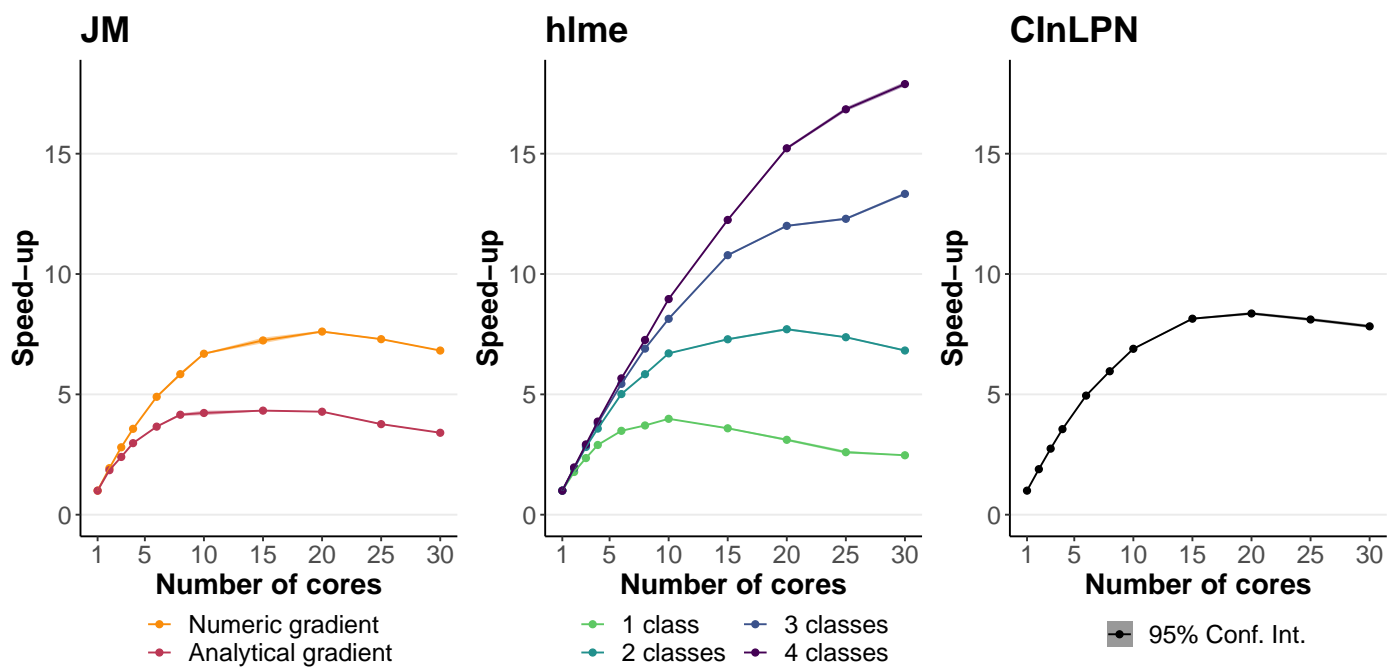

Figure 1: Speed up performances for the 3 different programs (JM, hlme, and CInLPN). Analytic and Numeric refer to the analytical and numerical computations of the gradient in JM. The number of parameters was 40 for JM; 10, 18, 26, 34 for hlme with 1, 2, 3, 4 classes, respectively; 27 for CInLPN.

between 2 and 10 to reach a maximal speed up slightly above 4 . With 15, 20, 25, or 30 cores, the performances were no more improved, the speed up showing even a slight reduction, probably due to the overhead. In contrast, when the program involved numerical computations of the gradient, the parallelization reduced the computation time by a factor of almost 8 at maximum. The better speed-up performances with a numerical gradient calculation were expected since the parallel loops iterate over more elements.

The second example, the latent class mixed model estimation (hlme), showed an improvement of the performances as the complexity of the models increased. The simple linear mixed model (one class model), like the joint models with analytical gradient, reached a maximum speed-up of 4 with 10 cores. The two-class mixed model with 18 parameters showed a maximum speed up of 7.71 with 20 cores. Finally, the 3 and 4-class mixed models reached speed-ups of 13.33 and 17.89 with 30 cores and might still be improved with larger resources.

The running time of the third program $(\mathrm{CInLPN})$ was also progressively reduced with the increasing number of cores reaching the maximal speed-up of 8.36 for 20 cores.

In these 7 examples, the speed up systematically reached almost 2 with 2 cores, and it remained interesting with 3 or 4 cores, although some variations in the speed-up performances began to be observed according to the complexity of the objective function computations. This highlights the benefit of the parallel implementation of MLA, even on personal computers. As the number of cores continued to increase, the speed-up performances varied a lot. Among our examples, the most promising situation was the one of the latent class mixed model (with a program in Fortran90) where 
the speed-up was up to 15 for 20 cores with the 4 class model.

\title{
Comparison with other optimization algorithms
}

\author{
Other Marquardt-Levenberg implementations
}

The Marquardt-Levenberg algorithm has been previously implemented in the context of nonlinear least squares problems in minpack.lm and nlmrt. We ran the examples provided in these two packages with marqLevAlg and compared the algorithms in terms of the final solution (that is, the residual sumof-squares) and runtime. Results are shown in the supplementary material. Our implementation reached exactly the same value as the two others but performed slower in these simple examples.

We also compared the sensitivity to initial values of marqLevAlg with minpack.lm using a simple example from minpack. $1 \mathrm{~m}$. We ran the two implementations of MLA on 100 simulated datasets, each one from 100 different starting points (see supplementary material). On the 10000 runs, marqLevAlg converged in $51.55 \%$ of the cases whereas the minpack.lm converged in $65.98 \%$ of the cases. However, 1660 estimations that converged according to nls.lm criteria were far from the effective optimum. This reduced the proportion of satisfying convergences with minpack. $1 \mathrm{~m}$ to $49.38 \%$ (so similar rate as marqLevAlg) but more importantly illustrated the convergence to saddle points when using classical convergence criteria. In contrast, all the convergences with marqLevAlg were closed to the effective solution thanks to its stringent RDM convergence criterion.

\section{Examples from the literature}

We tested our algorithm on 35 optimization problems designed by More et al. (1981) to test unconstrained optimization software and compared the marqLevAlg performances with those of several other optimizers, namely Nelder-Mead, BFGS, conjugate gradients (CG) implemented in the optim function, L-BFGS-B algorithm from optimParallel, and nlminb. Each problem consists of a function to optimize from given starting points. The results are presented in supplementary material in terms of bias between the real solution and the final value of the objective function. Our implementation of MLA converged in almost all the cases (31 out of 35), and provided almost no bias. Except for nlminb, which showed similar very good performances, the other algorithms converged at least once very far from the effective objective value. In addition, Nelder-Mead and CG algorithms converged only in approximately half of the cases.

\section{Example of complex optimization problem: Maximum Likelihood Estimation of a Joint model for longitudinal and time-to-event data}

Our implementation is particularly dedicated to complex problems involving many parameters and/or complex objective function calculation. We illustrate here its performances and compare them with other algorithms for the likelihood maximization of a joint model for longitudinal and time-to-event data, as an example of complex objective function optimization.

The JM package (Rizopoulos (2010)), dedicated to the maximum likelihood estimation of joint models, includes several optimization algorithms, namely the BFGS of optim function and an expectationmaximization (EM) technique internally implemented. It thus offers a nice framework to compare the reliability of MLA to find the maximum likelihood in a complex setting with the reliability of other optimization algorithms. We used in this comparison the prothro dataset described in the JM package and elsewhere (Skrondal and Rabe-Hesketh, 2004, Andersen et al. (1993)). It consists of a randomized trial in which 488 subjects were split into two treatment arms (prednisone versus placebo). Repeated measures of prothrombin ratio were collected over time as well as time to death. The longitudinal part of the joint model included a linear trajectory with time in the study, an indicator of the first measurement and their interaction with the treatment group. Correlated individual random effects on the intercept and the slope with time were also included. The survival part was a proportional hazard model adjusted for the treatment group as well as the dynamics of the longitudinal outcome either through the current value of the marker or its slope or both. The baseline risk function was approximated by B-splines with one internal knot. The total number of parameters to estimate was 17 or 18 (10 for the longitudinal submodel and 7 for the survival submodel, given that only the curent value of the marker or its slope or 8 for the survival model when both the current level and the slope were considered). The marker initially ranged from 6 to 176 (mean=79.0, sd=27.3).

To investigate the consistency of the results to different dimensions of the marker, we also considered cases where the marker was rescaled by a factor 0.1 or 10 . In these cases, the log-likelihood was 
rescaled a posteriori to the original dimension of the marker to make the comparisons possible. The starting point was systematically set at the default initial value of the jointModel function, which is the estimation point obtained from the separated linear mixed model and proportional hazard model.

In addition to EM and BFGS included in JM package, we also compared the MLA performances with those of the parallel implementation of the L-BFGS-B algorithm provided by the optimParallel package. Codes and dataset used in this section are available at https://github.com/VivianePhilipps/ marqLevAlgPaper.

MLA and L-BFGS-B ran on 3 cores. MLA converged when the three criteria defined in section 2.2.2 were satisfied with tolerance $0.0001,0.0001$, and 0.0001 for the parameters, the likelihood, and the RDM, respectively. BFGS and L-BFGS-B converged when the convergence criterion on the log-likelihood was satisfied with the square root of the tolerance of the machine $\left(\approx 10^{-8}\right)$. The EM algorithm converged when stability on the parameters or on the log-likelihood was satisfied with tolerance 0.0001 and around $10^{-8}$ (i.e., the square root of the tolerance of the machine), respectively.

\begin{tabular}{|c|c|c|c|c|c|c|c|}
\hline $\begin{array}{r}\text { Nature of } \\
\text { dependency }\end{array}$ & Algorithm & $\begin{array}{r}\text { Scaling } \\
\text { factor }\end{array}$ & $\begin{array}{r}\text { Rescaled log- } \\
\text { likelihood } \\
\end{array}$ & $\begin{array}{r}\text { Variation of } \\
\text { value (\%) }\end{array}$ & $\begin{array}{r}\text { Variation of } \\
\text { slope (\%) }\end{array}$ & $\begin{array}{r}\text { Number of } \\
\text { iterations }\end{array}$ & $\begin{array}{l}\text { Time in } \\
\text { seconds }\end{array}$ \\
\hline value & BFGS & 1 & -13958.55 & -3.73 & & 120 & 27.39 \\
\hline value & BFGS & 0.1 & -13957.91 & -0.01 & & 490 & 116.33 \\
\hline value & BFGS & 10 & -13961.54 & -9.28 & & 91 & 18.16 \\
\hline value & LBFGSB & 1 & -13958.41 & -3.56 & & 289 & 79.07 \\
\hline value & LBFGSB & 0.1 & -13957.69 & -0.11 & & 244 & 67.53 \\
\hline value & LBFGSB & 10 & error & & & & \\
\hline value & EM & 1 & -13957.91 & -0.29 & & 66 & 72.44 \\
\hline value & EM & 0.1 & -13957.72 & 0.14 & & 104 & 106.70 \\
\hline value & EM & 10 & -13957.94 & -0.59 & & 62 & 67.80 \\
\hline value & MLA & 1 & -13957.69 & -0.00 & & 7 & 34.37 \\
\hline value & MLA & 0.1 & -13957.69 & -0.00 & & 6 & 29.48 \\
\hline value & MLA & 10 & -13957.69 & -0.00 & & 17 & 75.48 \\
\hline slope & BFGS & 1 & -13961.41 & & -1.85 & 251 & 52.76 \\
\hline slope & BFGS & 0.1 & -13961.23 & & -1.37 & 391 & 87.61 \\
\hline slope & BFGS & 10 & -13980.90 & & -13.98 & 444 & 80.16 \\
\hline slope & LBFGSB & 1 & -13960.69 & & -0.15 & 266 & 60.29 \\
\hline slope & LBFGSB & 0.1 & -13960.70 & & -0.27 & 206 & 47.87 \\
\hline slope & LBFGSB & 10 & -13962.56 & & -2.87 & 823 & 182.20 \\
\hline slope & EM & 1 & -13960.69 & & 0.17 & 170 & 161.64 \\
\hline slope & EM & 0.1 & -13960.69 & & 0.02 & 208 & 196.68 \\
\hline slope & EM & 10 & -13960.70 & & 0.08 & 156 & 159.58 \\
\hline slope & MLA & 1 & -13960.69 & & -0.00 & 11 & 48.00 \\
\hline slope & MLA & 0.1 & -13960.69 & & -0.00 & 11 & 48.10 \\
\hline slope & MLA & 10 & -13960.69 & & 0.00 & 14 & 61.61 \\
\hline both & BFGS & 1 & -13951.60 & 15.97 & -28.17 & 164 & 37.83 \\
\hline both & BFGS & 0.1 & -13949.82 & 2.66 & -4.63 & 502 & 132.84 \\
\hline both & BFGS & 10 & -13965.25 & 40.31 & -95.26 & 52 & 10.48 \\
\hline both & LBFGSB & 1 & -13950.04 & -1.67 & 7.10 & 800 & 177.61 \\
\hline both & LBFGSB & 0.1 & -13949.42 & -0.01 & 0.38 & 411 & 93.31 \\
\hline both & LBFGSB & 10 & -13985.72 & 67.33 & -147.30 & 18 & 7.75 \\
\hline both & EM & 1 & -13949.82 & 4.10 & -7.22 & 159 & 186.69 \\
\hline both & EM & 0.1 & -13949.44 & 1.68 & -3.66 & 156 & 152.89 \\
\hline both & EM & 10 & -13950.46 & 10.67 & -16.31 & 142 & 220.07 \\
\hline both & MLA & 1 & -13949.42 & -0.00 & -0.00 & 10 & 49.91 \\
\hline both & MLA & 0.1 & -13949.42 & -0.00 & 0.00 & 10 & 51.63 \\
\hline both & MLA & 10 & -13949.42 & -0.00 & 0.00 & 24 & 121.69 \\
\hline
\end{tabular}

Table 4: Comparison of the convergence obtained by MLA, BFGS, LBFGSB, and EM algorithms for the estimation of a joint model for prothrobin repeated marker (scaled by $1,0.1$, or 10) and time to death when considering a dependency on the current level of prothrobin ('value'), the current slope ('slope'), or both ('both'). All the models converged correctly according to the algorithm outputs. We report the final log-likelihood rescaled to scaling factor 1 (for comparison), the percentage of variation of the association parameters ('value' and 'slope' columns) compared to the one obtained with the overall maximum likelihood with scaling 1 , the number of iterations and the running time in seconds.

Table 4 compares the convergence obtained using the four optimization methods when considering a pseudo-adaptive Gauss-Hermite quadrature with 15 points. All the algorithms converged correctly according to the programs except one with L-BFGS-B, which gave an error (non-finite value) during optimization. Although the model for a given association structure is exactly the same, some differences were observed in the final maximum log-likelihood (computed in the original scale of prothrombin ratio). The final log-likelihood obtained by MLA was always the same, whatever the outcome's scaling, showing its consistency. It was also higher than the one obtained using the three 
other algorithms, showing that BFGS, L-BFGS-B, and, to a lesser extent, EM did not systematically converge toward the effective maximum. The difference could go up to 20 points of log-likelihood for BFGS in the example with the current slope of the marker as the association structure. The convergence also differed according to outcome's scaling with BFGS/L-BFGS-B and slightly with EM, even though, in general, the EM algorithm seemed relatively stable in this example. The less stringent convergence of BFGS/L-BFGS-B and, to a lesser extent, of EM had also consequences on the parameters estimates as roughly illustrated in Table 4 with the percentage of variation in the association parameters of prothrombin dynamics estimated in the survival model (either the current value or the current slope) in comparison with the estimate obtained using MLA which gives the overall maximum likelihood. The better performances of MLA were not at the expense of the number of iterations since MLA converged in at most 22 iterations, whereas several hundreds of iterations could be required for EM or BFGS. Note, however, that one iteration of MLA is much more computationally demanding.

Finally, for BFGS, the problem of convergence was even more apparent when the outcome was scaled by a factor 10. Indeed, the optimal log-likelihood of the model assuming a bivariate association structure (on the current level and the current slope) was worse than the optimal log-likelihood of its nested model, which assumes an association structure only on the current level (i.e., constraining the parameter for the current slope to 0). We faced the same situation with the L-BFGS-B algorithm when comparing the log-likelihoods with a bivariate association and with an association through the current slope only.

\section{Concluding remarks}

We proposed in this paper a general-purpose optimization algorithm based on a robust MarquardtLevenberg algorithm. The program, written in $\mathrm{R}$ and Fortran90, is available in marqLevAlg $\mathrm{R}$ package. It provides a very nice alternative to other optimization packages available in $\mathrm{R}$ software such as optim, roptim (Pan, 2020), or optimx (Nash and Varadhan, 2011) for addressing complex optimization problems. In particular, as shown in our examples, notably the estimation of joint models, it is more reliable than classical alternatives (in particular EM, BFGS, and L-BFGS-B). This is due to the very good convergence properties of the Marquardt-Levenberg algorithm associated with very stringent convergence criteria based on the first and second derivatives of the objective function, which avoids spurious convergence at saddle points (Commenges et al., 2006).

The Marquardt-Levenberg algorithm is known for its very computationally intensive iterations due to the computation of the first and second derivatives. However, compared to other algorithms, it converges in a very small number of iterations (usually less than 30 iterations). This may not make MLA competitive in terms of running time in simple and rapid settings. However, the parallel computations of the derivatives can largely speed up the program and make it very competitive with alternatives in terms of running time in complex settings.

We chose in our implementation to rely on the RDM criterion, which is a very stringent convergence criterion. As it is based on the inverse of the Hessian matrix, it may cause non-convergence issues when some parameters are at the border of the parameter space (for instance, 0 for a parameter constrained to be positive). In that case, we recommend fixing the parameter at the border of the parameter space and running the optimization again on the rest of the parameters. In cases where the stabilities of the log-likelihood and of the parameters are considered sufficient to ensure satisfactory convergence, the program outputs might be interpreted despite a lack of convergence according to the $\mathrm{RDM}$, as is done for other algorithms that only converge according to the parameter and/or objective function stability.

As with any other optimization algorithm based on the steepest descent, MLA is a local optimizer. It does not ensure the convergence of multimodal objective functions toward the global optimum. In such a context, we recommend the use of a grid search which consists in running the algorithm from a grid of (random) initial values and retaining the best result as the final solution. We illustrate in supplementary material how this technique succeeds in finding the global minimum with the Wild function of the optim help page.

marqLevAlg is not the first optimizer to exploit parallel computations. Other R optimizers include a parallel mode, in particular stochastic optimization packages like DEoptim (Mullen et al., 2011), GA (Scrucca, 2017), rgenoud (Mebane, Jr. and Sekhon, 2011), or hydroPSO (Zambrano-Bigiarini and Rojas, 2020). We compared these packages, the local optimizer of optimParallel, and marqLevAlg for the estimation of the linear mixed model described in Section 2.4. For this specific problem marqLevAlg was the fastest, followed by optimParallel (results shown in supplementary files).

With its parallel implementation of derivative calculations combined with very good convergence properties of MLA, marq LevAlg package provides a promising solution for the estimation of complex statistical models in R. We have chosen for the moment to parallelize the derivatives, which is 
very useful for optimization problems involving many parameters. However, we could also easily parallelize the computation of the objective function when the latter is decomposed into independent sub-computations, as is the log-likelihood computed independently on the statistical units. This alternative is currently under development.

\section{Funding}

This work was funded by French National Research Agency [grant number ANR-18-CE36-0004-01 for project DyMES].

\section{Acknowlegdments}

The computing facilities MCIA (Mésocentre de Calcul Intensif Aquitain) at the Universite de Bordeaux and the Université de Pau et des Pays de l'Adour provided advice on parallel computing technologies, as well as computer time.

\section{Bibliography}

P. K. Andersen, O. Borgan, R. D. Gill, and N. Keiding. Statistical Models Based on Counting Processes. Springer, Paris, 1993. [p374]

D. Commenges, H. Jacqmin-Gadda, C. Proust, and J. Guedj. A Newton-Like Algorithm for Likelihood Maximization: The Robust-Variance Scoring Algorithm. arXiv:math/0610402, Dec. 2006. URL http://arxiv.org/abs/math/0610402. arXiv: math/0610402. [p366, 376]

T. V. Elzhov, K. M. Mullen, A.-N. Spiess, and B. Bolker. minpack.lm: R Interface to the LevenbergMarquardt Nonlinear Least-Squares Algorithm Found in MINPACK, Plus Support for Bounds, 2016. URL https://CRAN.R-project.org/package=minpack. Im. R package version 1.2-1. [p365, 366]

R. Fletcher. A modified Marquardt subroutine for non-linear least squares. 1971. Publisher: Theoretical Physics Division, Atomic Energy Research Establishment. [p366]

H. Joe and J. C. Nash. Numerical optimization and surface estimation with imprecise function evaluations. Statistics and Computing, 13(3):277-286, Aug. 2003. ISSN 1573-1375. doi: 10.1023/A: 1024226918553. URL https://doi.org/10.1023/A:1024226918553. [p365]

N. M. Laird and J. H. Ware. Random-Effects Models for Longitudinal Data. Biometrics, 38(4):963974, 1982. ISSN 0006-341X. doi: 10.2307/2529876. URL https: //www. jstor . org/stable/2529876. Publisher: [Wiley, International Biometric Society]. [p368]

K. Levenberg. A method for the solution of certain non-linear problems in least squares. Quarterly of Applied Mathematics, 2(2):164-168, July 1944. ISSN 0033-569X, 1552-4485. doi: 10.1090/qam/10666. URL http: //www. ams.org/qam/1944-02-02/S0033-569X-1944-10666-0/. [p365]

M. J. Lindstrom and D. M. Bates. Newton-Raphson and EM Algorithms for Linear MixedEffects Models for Repeated-Measures Data. Journal of the American Statistical Association, 83 (404):1014-1022, Dec. 1988. ISSN 0162-1459. doi: 10.1080/01621459.1988.10478693. URL https://doi.org/10.1080/01621459.1988.10478693. Publisher: Taylor \& Francis _eprint: https://doi.org/10.1080/01621459.1988.10478693. [p365]

D. W. Marquardt. An Algorithm for Least-Squares Estimation of Nonlinear Parameters. Journal of the Society for Industrial and Applied Mathematics, 11(2):431-441, June 1963. ISSN 0368-4245. doi: 10.1137/0111030. URL https://epubs.siam.org/doi/abs/10.1137/0111030. Publisher: Society for Industrial and Applied Mathematics. [p365, 366]

W. R. Mebane, Jr. and J. S. Sekhon. Genetic optimization using derivatives: The rgenoud package for R. Journal of Statistical Software, 42(11):1-26, 2011. URL http: //www. jstatsoft. org/v42/i11/. [p376]

J. J. More, B. S. Garbow, and K. E. Hillstrom. Testing unconstrained optimization software. ACM Trans. Math. Software, 7(1), 3 1981. doi: 10.1145/355934.355936. URL https://www. osti.gov/biblio/ 6256067. [p374] 
K. Mullen, D. Ardia, D. Gil, D. Windover, and J. Cline. DEoptim: An R package for global optimization by differential evolution. Journal of Statistical Software, 40(6):1-26, 2011. URL http: //www. jstatsoft. org/v40/i06/. [p376]

J. C. Nash. nlmrt: Functions for Nonlinear Least Squares Solutions, 2016. URL https://CRAN. R-project. org/package=nlmrt. R package version 2016.3.2. [p365, 366]

J. C. Nash and R. Varadhan. Unifying optimization algorithms to aid software system users: optimx for R. Journal of Statistical Software, 43(9):1-14, 2011. URL http: //www. jstatsoft. org/v43/i09/. [p365, 376]

Y. Pan. roptim: General Purpose Optimization in R using C++, 2020. URL https: //CRAN. R-project.org/ package=roptim. R package version 0.1.5. [p376]

M. Prague, A. Diakite, and D. Commenges. Package 'marqLevAlg' - Algorithme de LevenbergMarquardt en R : une Alternative à 'optimx' pour des Problèmes de Minimisation. In 1ères Rencontres R, Bordeaux, France, July 2012. URL https://hal . archives-ouvertes. fr/hal-00717566. [p365]

M. Prague, D. Commenges, J. Guedj, J. Drylewicz, and R. Thiébaut. Nimrod: A program for inference via a normal approximation of the posterior in models with random effects based on ordinary differential equations. Computer methods and programs in biomedicine, 111(2):447-458, 2013. [p366]

C. Proust and H. Jacqmin-Gadda. Estimation of linear mixed models with a mixture of distribution for the random effects. Computer Methods and Programs in Biomedicine, 78(2):165-173, May 2005. ISSN 0169-2607. doi: 10.1016/j.cmpb.2004.12.004. [p365]

C. Proust-Lima, M. Séne, J. M. Taylor, and H. Jacqmin-Gadda. Joint latent class models for longitudinal and time-to-event data: A review. Statistical methods in medical research, 23(1):74-90, Feb. 2014. ISSN 0962-2802. doi: 10.1177/0962280212445839. URL https://www.ncbi.nlm.nih.gov/pmc/articles/ PMC5863083/. [p370]

C. Proust-Lima, V. Philipps, and B. Liquet. Estimation of Extended Mixed Models Using Latent Classes and Latent Processes: The R Package lcmm. Journal of Statistical Software, 78(1):1-56, June 2017. ISSN 1548-7660. doi: 10.18637/jss.v078.i02. URL https://www. jstatsoft.org/index.php/jss/article/ view/v078i02. Number: 1. [p370]

D. Rizopoulos. JM: An R package for the joint modelling of longitudinal and time-to-event data. Journal of Statistical Software (Online), 35(9):1-33, July 2010. ISSN 15487660. doi: 10.18637/jss.v035.i09. URL https://repub.eur.n1/pub/89690/. [p370, 371,374]

L. Scrucca. On some extensions to GA package: hybrid optimisation, parallelisation and islands evolution. The R Journal, 9(1):187-206, 2017. URL https: // journal . r-project.org/archive/2017/ RJ-2017-008. [p376]

A. Skrondal and S. Rabe-Hesketh. Generalized Latent Variable Modeling: Multilevel, Longitudinal, and Structural Equation Models. CRC Press, May 2004. ISBN 978-0-203-48943-7. [p374]

B. O. Taddé, H. Jacqmin-Gadda, J.-F. Dartigues, D. Commenges, and C. Proust-Lima. Dynamic modeling of multivariate dimensions and their temporal relationships using latent processes: Application to Alzheimer's disease. Biometrics, n/a(n/a), 2019. ISSN 1541-0420. doi: 10.1111/ biom.13168. URL https://onlinelibrary.wiley.com/doi/abs/10.1111/biom.13168. _eprint: https://onlinelibrary.wiley.com/doi/pdf/10.1111/biom.13168. [p370, 372]

S. Theussl, F. Schwendinger, and H. W. Borchers. CRAN task view: Optimization and mathematical programming, 2014. URL https: //cran. r-project.org/view=0ptimization. Version 2020-05-21. [p365]

M. Zambrano-Bigiarini and R. Rojas. hydroPSO: Particle Swarm Optimisation, with Focus on Environmental Models, 2020. URL https://CRAN.R-project.org/package=hydroPSO. R package version 0.5-1. doi:10.5281/zenodo.1287350. [p376]

\author{
Viviane Philipps \\ Inserm, Bordeaux Population Health Research Center, UMR 1219, \\ Univ. Bordeaux, F-33000 Bordeaux, France \\ 146 rue Léo Saignat \\ 33076 Bordeaux Cedex \\ France \\ E-mail: viviane.philipps@u-bordeaux.fr
}


Boris P. Hejblum

Inserm, Bordeaux Population Health Research Center, UMR 1219, Inria BSO SISTM,

Vaccine Research Institute (VRI), F-94000 Créteil, France

Univ. Bordeaux, F-33000 Bordeaux, France

146 rue Léo Saignat

33076 Bordeaux Cedex

France

E-mail: boris. hejblum@u-bordeaux.fr

Mélanie Prague

Inserm, Bordeaux Population Health Research Center, UMR 1219,

Inria BSO SISTM,

Vaccine Research Institute (VRI), F-94000 Créteil, France

Univ. Bordeaux, F-33000 Bordeaux, France

146 rue Léo Saignat

33076 Bordeaux Cedex

France

E-mail: melanie.prague@inria.fr

Daniel Commenges

Inserm, Bordeaux Population Health Research Center, UMR 1219, Inria BSO SISTM,

Univ. Bordeaux, F-33000 Bordeaux, France

146 rue Léo Saignat

33076 Bordeaux Cedex

France

E-mail: daniel.commenges@u-bordeaux.fr

Cécile Proust-Lima

Inserm, Bordeaux Population Health Research Center, UMR 1219,

Univ. Bordeaux, F-33000 Bordeaux, France

146 rue Léo Saignat

33076 Bordeaux Cedex

France

E-mail: cecile.proust-lima@inserm.fr 